\title{
Stakeholder Attitudes on Carbon Capture and Storage - an international comparison
}

\author{
Filip Johnsson ${ }^{\mathrm{a}}$, David Reiner ${ }^{\mathrm{b}}$, Kenshi Itaoka ${ }^{\mathrm{c}}$, Howard Herzog ${ }^{\mathrm{d}}$ \\ ${ }^{a}$ Department of Energy and Environment, Energy Conversion, Chalmers University of Technology, SE-412 96 Göteborg, Sweden \\ ${ }^{b}$ Judge Business School, University of Cambridge,Trumpington Street, Cambridge, UK, CB2 1AG \\ ${ }^{c}$ Mizuho Information and Research Institute, Tokyo 101-8443, Japan \\ ${ }^{d}$ Massachusetts Institute of Technology, Cambridge, Massachusetts 02139-04307, USA
}

Elsevier use only: Received date here; revised date here; accepted date here

\begin{abstract}
This paper presents results from a survey on stakeholder attitudes towards Carbon Capture and Storage (CCS). The survey is the first to make a global comparison across three major regions; USA, Japan, and Europe. The 30-question survey targeted individuals working at stakeholder organizations that seek to shape, and will need to respond to, policy on CCS, including electric utilities, oil \& gas companies, CO2-intensive industries and non-governmental organizations (NGOs).

The results show generally small differences across the regions and between the different groups of stakeholders. All believed that the challenge of significant reductions in emissions using only current technologies was severe. There is a widespread belief both that renewable technologies such as solar power and CCS will achieve major market entry into the electricity sector within the next 10 to 20 years, whereas there is more skepticism about the role of hydrogen and especially nuclear fusion in the next 50 years. All groups were generally positive towards renewable energy. Yet, there were some notable areas of disagreement in the responses, for example, as expected, NGOs considered the threat of climate change to be more serious than the other groups. North Americans respondents were more likely to downplay the threat compared to those of the other regions. The Japanese were more concerned about the burden that would be placed on industry in the coming decade as a result of emissions constraints and NGOs were more likely to believe that the burden would be light or very light. NGOs believed CCS to be far more attractive than nuclear fusion power but much less than renewables. As expected, the risk for leakage from reservoirs was ranked number one of the risk options given.
\end{abstract}

(C) 2008 Elsevier Ltd. All rights reserved

Keywords: Stakeholder perspectives; carbon dioxide and storage technologies;

\section{Introduction}

Different technological options and measures for reducing anthropogenic $\mathrm{CO}_{2}$ emissions including using less energy (energy conservation measures, increasing energy efficiency), shifting fuels (coal to gas, increased use of renewables and nuclear power) and capturing and storing $\mathrm{CO}_{2}$ (land use change and forestation, capturing $\mathrm{CO}_{2}$ from large point sources). Widespread introduction of these technologies and measures require research and development to improve performance, reliability and efficiency as well as supporting policies. No matter how promising an 
option is from a technological and economic perspective, it has to be socially acceptable if implementation is to be successful. Thus, it is necessary to investigate not only technical and economic barriers which must be overcome for successful implementation, but also potential social and political barriers. This is especially true for technologies which are new and have not yet been implemented at scale. $\mathrm{CO}_{2}$ Capture and Storage (CCS) is one such example. CCS is also important in that it is expected to significantly contribute to reaching climate targets from 2020 to 2050, i.e. in the medium term. Yet, there is not yet a large scale demonstration project of CCS where the entire chain from capture, and transport to storage, is demonstrated (especially nothing which is visible to the public). This is rather unique in the sense that most other technologies and measures which are foreseen to significantly contribute to the transformation of the energy system up to year 2050 are used or have been demonstrated at significant scale. Whether this is good or not when it comes to make CCS socially acceptable is not clear, but it should be of great importance to map and understand public as well stakeholder attitudes towards CCS. This study is part of such a mapping and focus on stakeholders.

The literature on public acceptance of CCS is relatively sparse, especially before 2005 (IPCC, 2005). Several more recent surveys of societal attitudes have focused on public opinion surveys (e.g. de Best-Waldhober et al., 2006, Reiner et al., 2006) and still fewer stakeholder surveys have been conducted. Shackley et al. (2007) investigate stakeholder perceptions on CCS in Europe and their study also share some questions with this work. Schackley et al. found that a majority of the respondents considered that CCS is 'definitely' or 'probably necessary' to achieve deep reductions in CO2 emissions between now and 2050 in their own country. This is in agreement with a survey by Ramírez et al. (2007) which sought to define criteria for CCS as part of the development of a sustainable energy system ${ }^{1}$.

This study concerns social and political barriers with emphasis on CCS in relation to other technologies and measures. The study is made among individuals working at stakeholder organizations that seek to shape, and will need to respond to, policy on CCS, including electric utilities, oil \& gas companies, $\mathrm{CO}_{2}$-intensive industries and non-governmental organizations (NGOs). The aim is to identify, study, and address non-technical issues associated with CCS from fossil-fired energy production, and to provide guidance to decision makers. The study is a cooperative effort between Chalmers University of Technology (Sweden), MIT (USA), University of Cambridge (UK), and Mizuho Information and Research Institute (Japan), where each institution performed corresponding surveys in their own region.

\section{Method}

The work is based on a questionnaire, sent out in 2006, which consisted of 31 identical questions in all regions North America, Europe and Japan). For all questions reported here, the respondents were given multiple choice questions. The person receiving the questionnaire was asked to answer it on an individual basis, i.e. based on her/his personal opinion and personal knowledge. Consequently, the responses do not necessarily represent the official opinion of each stakeholder organization. In all, just over 140 individuals responded to the questionnaire, thus the results should only be seen as indicative. Results are generally presented by the number of all respondents choosing each possible answer. When possible, this is complemented with qualitative observations regarding similarities and differences between individuals working at each type of stakeholder organization.

\section{Results}

This section exemplifies results from the survey and covers questions related to general issues on climate change and then reports on 10 (out of 19) of the questions related to CCS.

\subsection{General Background on Climate Change}

In all, the stakeholders were asked seven questions regarding climate change and potential solutions. The first three questions concerned the seriousness of climate change compared to other problems in society and the impacts of regulation and policies related to climate change. The other four questions dealt with different aspects of reducing CO2 emissions and how climate change issues are handled within the stakeholders' organizations.

\footnotetext{
${ }^{1}$ Ramírez et al. (2008), p141: “The majority of respondents (58\% total sample; 63\% Dutch respondents) considered CCS an important option because it creates greater lead-time to develop cost-effective renewables.”
} 


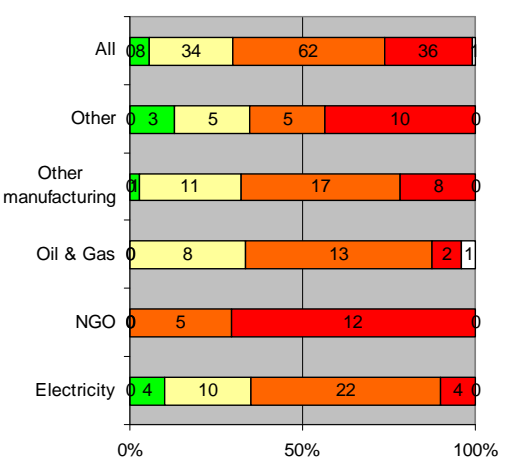

a.

Figure 1. Responses to the question "How serious do you consider the threat of climate change to be relative to other problems facing society (such as starvation, poverty, crime etc)?” The numbers on each bar illustrate the number of stakeholders choosing this alternative answer. a. division with respect to stakeholder group (all regions), b. division between regions.

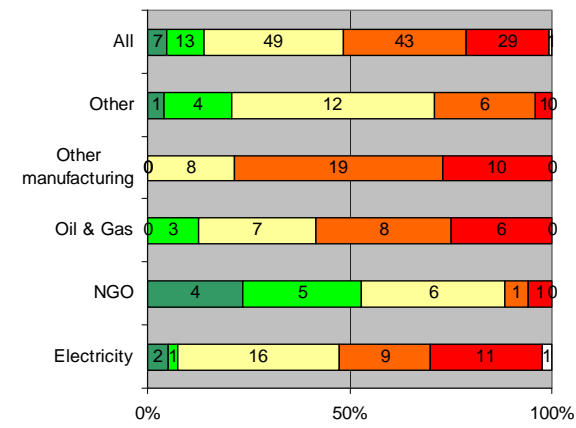

a.
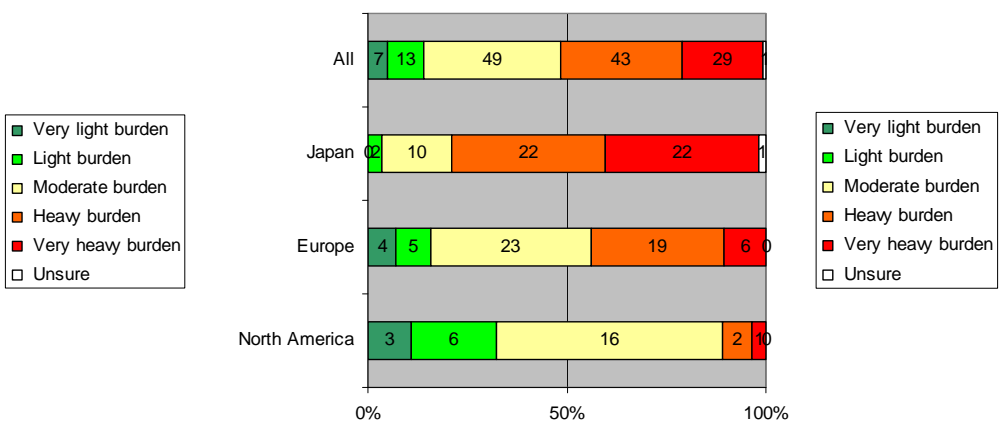

b.

Figure 2. Responses to the question "How much of a burden do you expect climate change policies to impose on businesses over the next decade?" The numbers on each bar illustrate the number of stakeholders choosing this alternative answer. a. division with respect to stakeholder group (all regions), b. division between regions.
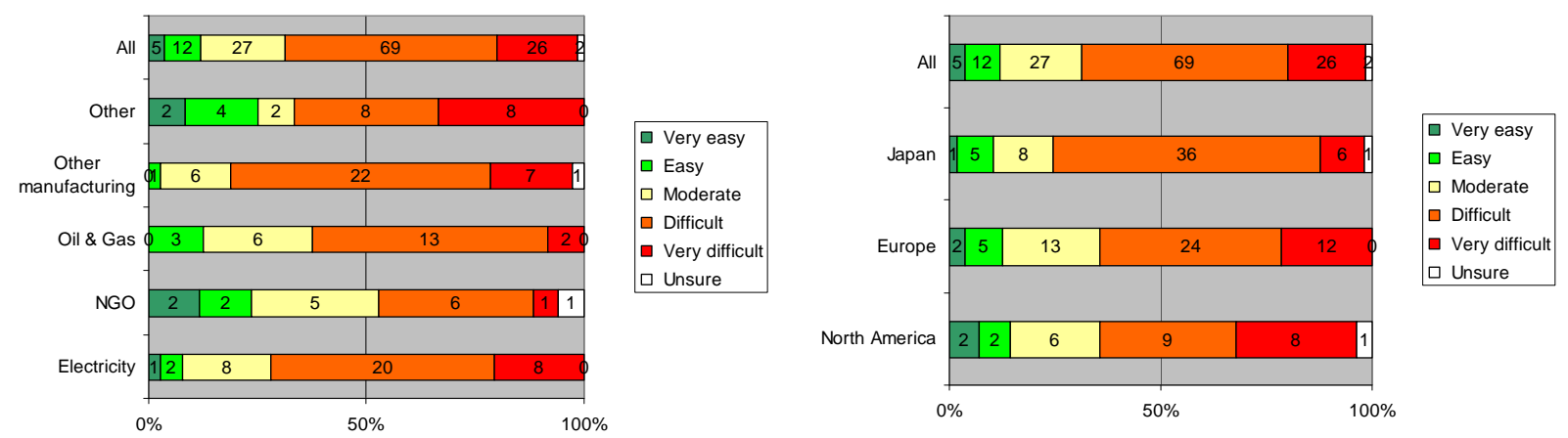

a.

b.

Figure 3. Responses to the question "How difficult do you think it will be to reduce emissions significantly using current best available approaches (such as conservation, efficiency, wide-scale deployment of renewables, fuel switching to less carbon-intensive fuels, increased use of nuclear power)?” The numbers on each bar illustrate the number of stakeholders choosing this alternative answer. a. division with respect to stakeholder group (all regions), b. division between regions. 
For most of these questions, the results were rather similar across the regions and between the different groups of stakeholders. There were, however, some notable areas of disagreement as can be seen from Figure 1 . As an example, NGOs considered, as expected, the threat of climate change to be more serious (Figure1a) than other groups. Also, North American respondents were more likely to downplay the threat of climate change compared to those of the other regions, although they also expressed a greater polarization in opinions (Figure 1b).

From Figure 2a it can be seen that compared with the other groups, NGOs were more likely to believe that the burden that would be placed on industry in the coming decade as a result of emissions constraints would be light or very light whereas the Japanese were more concerned about the burden. Figure 3 shows the belief in current available technologies for achieving significant reductions in CO2 emissions. Although a majority considers it to be difficult or very difficult to significantly cut CO2 emissions by means of currently available technologies, NGOs are clearly more optimistic in this respect (Figure 3a). In response to the question "If emissions are reduced, which do you think will be the major driver in reducing emissions - advances in technology or changing individual behavior?” all stakeholder groups across all regions gave answers in favor of technologies over change in lifestyle. Yet, the largest fraction of answers was for a combination of technologies and changed lifestyle.

In summary, it can be concluded that all groups in all regions consider the climate change to be a real threat but that it can be avoided if action is taken and that there are technologies available to solve the problem. However, the respondents seem to have less faith in that there will be a strong enough policy to meet the climate targets.

\subsection{Future of Carbon Capture and Storage (CCS)}

In this paper, the results from the following 10 questions are discussed (choices for answers in brackets):

Q1. Which of the following options give the best description of the relationship between the effect of CCS on electricity prices faced by consumers and penetration of other low-carbon alternative sources of energy such as renewables or nuclear? (Increased adoption of CCS will encourage/not influence/discourage/unsure renewables/ nuclear energy)

Q2. How would you characterize the role that CCS plays in the current national climate change debate in your country? (major, significant, minor, negligible, non-existent, unsure)

Q3. Do you believe that the role of CCS is increasing or decreasing in the national climate change debate in your country? (Increasing substantially, increasing slightly, staying the same, decreasing slightly, decreasing substantially, unsure)

Q4. In general terms, how would you compare the following electric power sector technologies to fossil-fired plants with carbon capture and storage? - Natural gas turbines, Conventional coal power, Hydropower, Wind turbines, Nuclear power, Biomass/bioenergy, Solar power, Nuclear fusion, (Much more preferable than CCS, More preferable than CCS, Similar to CCS, Less preferable than CCS, Much less preferable than CCS, Unsure)

Q5. When do you think that large-scale entry of the following technologies in the electric power sector is likely? CCS, Solar energy, Fuel cells, Hydrogen power, Nuclear fusion, Tidal power, (Within the next 10 years, In 20 years, In 50 years, Never)

Q6. Which of the following would you consider to be to be most significant concerns that would discourage widescale penetration of CCS? (choices grouped into the following areas: Social acceptability, Siting, Economics, Technical and Institutional design, Other)

Q7. What would you think is the current attitude among the public toward CCS? (Very positive, Moderately positive, Ambivalent, Moderately skeptical, Hostile, Largely ignorant, Unsure)

Q8. When would you expect that the public would begin to understand the issues associated with CCS? (Next few years, Next few decades, Only if CCS becomes controversial, Only when confronted with a local siting question, Never, Unsure)

Q9. Does your organization currently have an official position on CCS? (Yes, it is positive toward CCS, Yes, it is neutral toward CCS, Yes, it is negative toward CCS, No, Discussions underway, Unsure) 
Q10. Which do you believe to be the major sources of risk for CCS? (Accidents in transport and handling, Injection at storage sites, Leakage from reservoirs, Seismic activity, Other, None of the above, Unsure).

The first question (Q1) relates to the argument sometimes expressed against CCS; that CCS will discourage investments in renewable technologies. As can be seen from Figure 4, this opinion exists in all of the groups investigated. Yet, the other point of view that increased adoption of CCS will encourage penetration of renewable is significantly more common. Only among NGOs both alternatives give similar response rate. Among NGOs one would perhaps have expected a majority of the answers to be "CCS will discourage renewables” (cf. e.g. Ramírez et al, 2008). The reason this is not the case may be that the question links the effects of CCS to the electricity price (i.e. the respondents are likely to have linked the introduction of CCS to an increased electricity price which in turn would make renewables more competitive). As for Q1, there were no clear differences between the regions.

With respect to the role of CCS in the national climate debate (Q2), in all regions and for all groups more respondents considered it as playing a small or very small role compared to those who considered it as playing a large or very large role. As shown in Figure 5, the more optimistic assessments were more common in Japan and North America than in Europe. However, the climate change debate is constantly changing and it might be expected that results would differ if the questionnaire had been sent out today. Also, an overwhelmingly majority of respondents considered the role of CCS in the national debate to be increasing (Q3).

Among the public as well as among stakeholders and politicians there have always been strong opinions on which technologies and measures to be preferred choice in transforming the energy system. Arguments relate not only to emissions but also to security of supply and siting issues. As for preferences of technologies, Figure 6 exemplifies results from Q4, i.e. CCS compared to wind power and nuclear power (fission). As can be seen wind power is preferred over CCS in almost all groups whereas CCS is rather similar to nuclear power, but with differences between the groups. For electricity companies where one can expect significant knowledge on CCS, CCS is clearly preferable to nuclear, a result similar to that obtained by Gough (2008). Given their longstanding opposition, NGOs, unsurprisingly, clearly prefer CCS over nuclear power. In general renewable energy was preferred over CCS. Question Q4 did not relate the technologies to costs or ability to contribute to CO2 reductions which if included in question may of course have given a different picture. A previous study by the authors (Reiner et al. 2006) polled the public in the same four regions as in this study. A majority of the public strongly preferred renewable-based technologies over fossil fuelled technologies and nuclear power. As expected the knowledge of CCS was very low. The results of this work show that the stakeholders are better informed on CCS than the public. Yet, some of the questions (not reported in this paper) also indicated a lack of knowledge on what is meant by EOR and what different storage options are available.

In response to the question of when large-scale entry of the different technologies (Q5) can be expected for CCS, solar energy and fuel cells, a majority of the respondents expected entry in 10 or in 20 years with answers about equally distributed between these two choices (except for Japan where more respondents stated "in 20 years" than “in 10 years”). Figure 7a illustrates the responses for CCS. For Hydrogen, expectations were shifted towards even longer time horizons of 20 or 50 years, with European respondents being more pessimistic and the Japanese more optimistic. With respect to stakeholder group preferences, the Oil and Gas industry is most optimistic on Hydrogen. Only around $10 \%$ of respondents believed there will be no future for CCS ("Never" on entry). As seen from Figure 7b, the largest fraction of respondents believed it will take 50 years until there is a major market entry for nuclear fusion. This can perhaps be expected, but as can be seen there is almost an equally large fraction of stakeholders who think that nuclear fusion will never come to market. This may be due to the fact that although CCS is not yet demonstrated at large scale, it is, to a large extent, based on commercially proven technologies, i.e. each part of the chain capture, transport and storage has to some extent been demonstrated or can be envisioned. This is not the case for nuclear fusion and it is also likely that the "never" answers to some extent can be interpreted as "no knowledge” since it was not possible to chose "unsure” as an answer (and that Q4 on technology choice gave a large part of answers in the "unsure” group for nuclear fusion). 


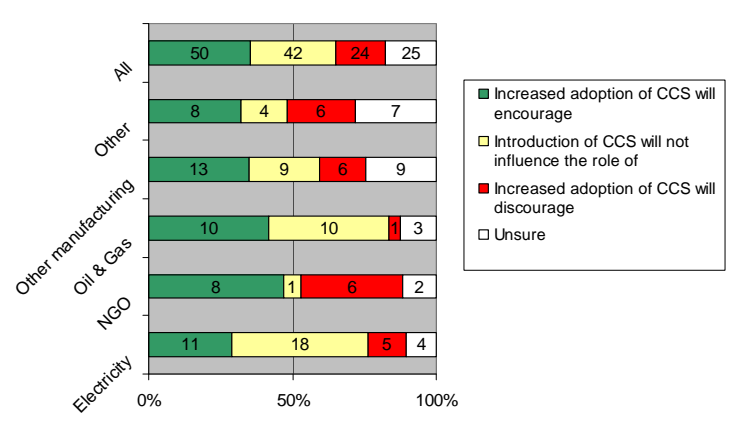

Figure 4. Responses to question Q1 (CCS will discourage/ encourage renewables) for the different groups investigated.

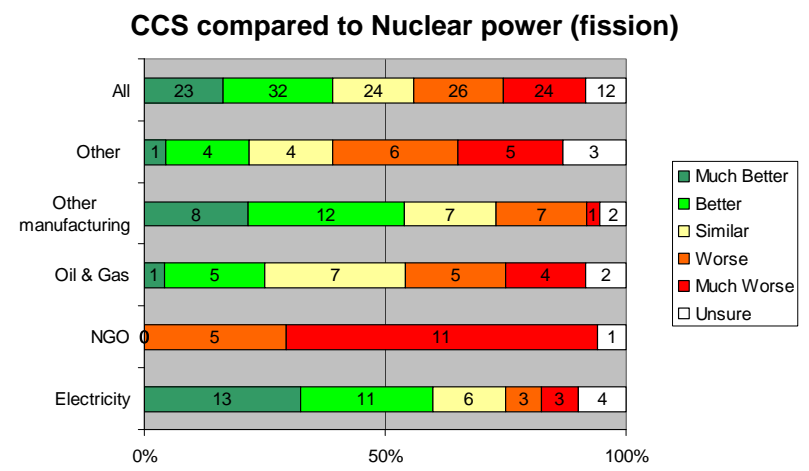

a.

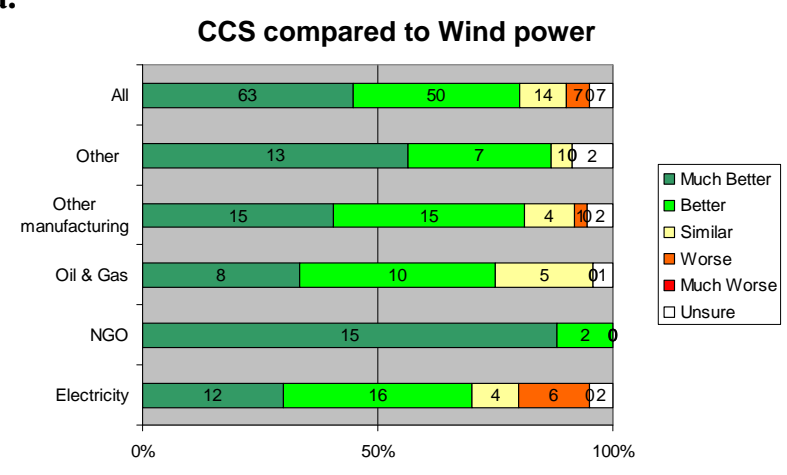

c.

Figure 6. Responses to question Q4 (CCS compared to other technologies). Examples compare CCS to nuclear (a, b) and wind (c, d).

As for Q6 on which would be to be most significant concerns that would discourage wide-scale penetration of CCS, economic viability, finding suitable storage, acceptability to local publics, capital costs and acceptability to a wider public were the most common answers. All these alternatives obtained support of around 7 to $10 \%$. Even fewer respondents believed acceptability to business community, equity or fairness in siting, accounting and securing credit for activities to limit implementation of CCS (generally less than 5\% of answers).

From Figure 8 (Q7) it can be seen that, as expected, a majority of all stakeholder groups (in all regions), typically 60 to $70 \%$, thought the public to be largely ignorant on CCS.. Since a majority of respondents considered the role of CCS in debate to be increasing (Q3), one could expect that if poll had been carried out today, the stakeholders would probably have thought public to be more informed. However, as seen from Figure 9 the answers from Q8 indicate that a majority of respondents think that the public will begin to understand the issues associated with CCS only if it 


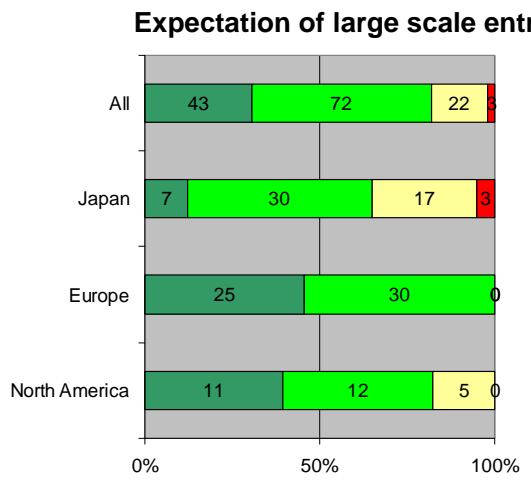

a.

Figure 7. Responses to question Q5 (When large scale entry of different technologies to be expected). Examples for a CCS and $\mathbf{b}$ Nuclear fusion.

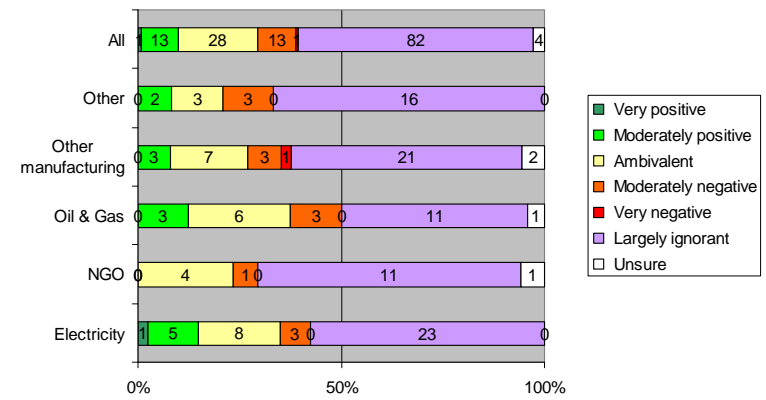

Figure 8. Responses to question $Q 7$ (Stakeholders belief in attitude among the public toward CCS).
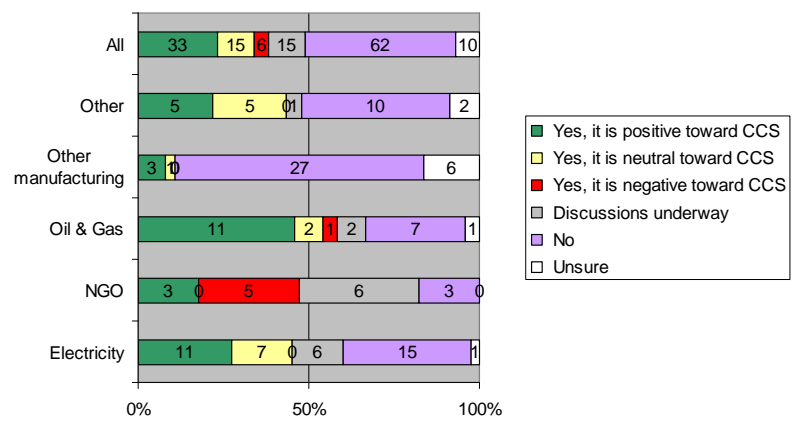

a.

Figure 10. Responses to question Q9: If the organization of the respondent has an official position on CCS. a. division with respect to stakeholder groups and $\mathbf{b}$. for the different regions.

becomes controversial or when confronted with a local siting question (45 to 55\% with about equal split between these two options). Those who believed that the public would begin to understand CCS issues within the next few years ranged from about 25\% (in Japan) to around 40\% (in North America). There are also minimal differences between stakeholder groups, except that Oil \& Gas industry respondents were somewhat more optimistic, with 50\% providing the answer that they think the public will begin to understand CCS within next few years. In summary, there is a general concern that the public is not informed on CCS (also a conclusion made by Ramírez et al, 2008) which is also confirmed by polls on CCS (e.g. Reiner et al., 2006). Here, follow up studies on stakeholder and public attitudes are important to see if stakeholder concerns decrease and/or public knowledge increases over time. 
Figure 10 shows that few organizations have an official position on CCS. As might be expected, NGOs and companies in the electricity and oil \& gas industries are more likely to have a clear position on CCS than other stakeholders. Figure 10a shows that NGOs differ from the other groups of stakeholder organizations by having the largest share of clearly negative positions on CCS. There are also some regional differences in the answers to Q9. From the answers (Figure 10b), it is evident that a larger share of stakeholder organizations in North America than in Europe and Japan has a clear on position on CCS.

With respect to the risks of CCS (Q10) the most common concern was, as expected, the risk for leakage from reservoirs (about 40\% in total) followed by seismic activity (25\%) and risks associated with transport and handling (around 20\%). There was rather little difference in ranking of risks between the groups (but note that Q10 concerns ranking of risks and not if risks are large or small).

Finally there was a strong consensus across both regions and stakeholder groups that more stringent regulations on carbon dioxide emissions will lead to advances in CCS rather than that advances in CCS leading to more stringent emission standards (question not presented in this paper). This point to the importance of imposing a cost associated with emitting $\mathrm{CO}_{2}$ in order to obtain penetration of technologies which reduce $\mathrm{CO} 2$ emissions, including CCS. Without sufficient carbon cost, continued use of fossil fuels without $\mathrm{CO}_{2}$ capture would make it impossible to meet aggressive scenarios aimed at reducing emissions and stabilizing greenhouse gas concentrations..

\section{Conclusions}

A majority of stakeholders surveyed believed the threat of climate change is one of the most severe threats society is currently facing and that significant efforts are required to solve this problem. In addition, most respondents think it will be a great challenge to solve the problem using available technologies. There is a widespread belief both that renewable technologies such as solar power and CCS will achieve major market entry into the electricity sector within the next 10 to 20 years. There was also an agreement on both the obstacles facing deployment of CCS and the opportunities driving CCS forward.

There were some areas of disagreement in the responses such as the seriousness of the climate change threat and the burden that would be placed on industry in the coming decade as a result of emissions constraints. NGOs believed CCS to be far more attractive than nuclear (fission) power but much less than renewables.

\section{Acknowledgements}

This work was supported by the Alliance for Global Sustainability (AGS), AIST (National Institute of Advanced Industrial Science and Technology) of Japan, and the MIT Carbon Sequestration Initiative. Funding is also from the AGS project "Pathways to Sustainable European Energy Systems". Mattias Bisaillon (Sweden) is greatly acknowledged for assisting in the analysis.

\section{References}

de Best-Waldhober, M., Daamen, D., Damen, K., Faaij, A., Centre for Energy and Environmental Studies, Faculty of Social Sciences, Leiden, University and Copernicus Institute, Utrecht University, The Netherlands, 2006.

Gough, C., Int. J. Greenhouse Gas Control 2, 2008, pp 155-168.

IPCC Special Report on Carbon Dioxide Capture and Storage (Cambridge: Cambridge University Press)

Ramírez, A., Hoogwijk, M., Hendriks, C., Faaij, A., Int. J. Greenhouse Gas Control 2, 2008, pp136-154.

Reiner, D.M., Curry, T.E., De Figueiredo, M.A., Herzog, H.J., Ansolabehere, S.D., Itaoka, K., Johnsson, F., Odenberger, M., Environmental Science and Technology 40, 2006, pp. 2093-2098

Shackley, S., Waterman, H., Godfroij, P., Reiner, D., Anderson, D., Draxlbauer, K., Flach, T., Energy Policy 35, 2007, pp. 5091-5108. 